\title{
BANACH FUNCTION ALGEBRAS WITH DENSE INVERTIBLE GROUP
}

\author{
H. G. DALES AND J. F. FEINSTEIN
}

(Communicated by N. Tomczak-Jaegermann)

\begin{abstract}
In 2003 Dawson and Feinstein asked whether or not a Banach function algebra with dense invertible group can have a proper Shilov boundary. We give an example of a uniform algebra showing that this can happen, and investigate the properties of such algebras. We make some remarks on the topological stable rank of commutative, unital Banach algebras. In particular, we prove that $\operatorname{tsr}(A) \geq \operatorname{tsr}\left(C\left(\Phi_{A}\right)\right)$ whenever $A$ is approximately regular.
\end{abstract}

\section{INTRODUCTION}

Let $A$ be a commutative, unital Banach algebra. The character space of $A$ is denoted by $\Phi_{A}$, as in [8]. For $a \in A$, we denote the Gel'fand transform of $a$ by $\widehat{a}$. We say that $\Phi_{A}$ contains analytic structure if there is a continuous injection $\tau$ from the open unit disk $\mathbb{D}$ to $\Phi_{A}$ such that, for all $a \in A, \widehat{a} \circ \tau$ is analytic on $\mathbb{D}$. In [16], Stolzenberg gave a counter-example to the conjecture that, whenever a uniform algebra has proper Shilov boundary, its character space must contain analytic structure (see also [17, Theorem 29.19], [19] and [1]). Cole gave an even more extreme example in his thesis [3], where the Shilov boundary is proper and yet every Gleason part is trivial. It is elementary to show that the invertible group of $A$ cannot be dense in $A$ whenever $\Phi_{A}$ contains analytic structure. The converse is false, as is shown by the uniform algebra $C(\overline{\mathbb{D}})$ of all continuous, complex-valued functions on $\overline{\mathbb{D}}$. Thus the requirement that the invertible group be dense is strictly stronger than the non-existence of analytic structure in the character space of the algebra. This leads to a new conjecture: that no uniform algebra with dense invertible group can have a proper Shilov boundary. This was raised (as an open question) by Dawson and the second author in [9].

Here, we first modify the example of Stolzenberg to show, in $\S 2$, that this new conjecture is also false. Our example is of the form $P(X)$ for a compact set $X \subseteq \mathbb{C}^{2}$. In our example, the Shilov boundary is proper, and yet there is a dense set of functions in the algebra $P(X)$ whose spectra have empty interiors. It is clear that this latter condition is sufficient for the invertible group to be dense in the algebra.

In fact, this sufficient condition is also necessary for the invertible group to be dense, as is shown in [10] and [7, Corollary 1.10]. Note, however, that it is not true that a function in the closure of the invertible group must have a spectrum whose

Received by the editors December 1, 2005 and, in revised form, October 23, 2006 and December 20, 2006.

2000 Mathematics Subject Classification. Primary 46J10. 
interior is empty. For example, the invertibles are dense in the uniform algebra $C(\mathbb{I})$, where $\mathbb{I}=[0,1]$, but there are functions in $C(\mathbb{I})$ whose range (and spectrum) is the unit square.

In $\S 3$, we shall obtain a new result about the topological stable rank (to be defined below) of approximately regular commutative, unital Banach algebras; we note that a commutative, unital Banach algebra has dense invertibles if and only if it has topological stable rank equal to 1.

We recall some standard notation and results. For the basic theory of commutative, unital Banach algebras, see, for example, [8], [12], and [17].

In our terminology, a compact space is a non-empty, compact, Hausdorff topological space. We denote by $\operatorname{dim} X$ the covering dimension of $X$; see [14] for details of many equivalent definitions of $\operatorname{dim} X$.

Let $X$ be a non-empty set. The uniform norm on $X$ is denoted by $|\cdot|_{X}$. Now let $X$ be a compact space. The algebra of continuous, complex-valued functions on $X$ is denoted by $C(X)$.

Let $A$ be a unital algebra. Then $\operatorname{Inv} A$ denotes the invertible group of $A$. Now suppose that $A$ is a unital Banach algebra. Then we say that $A$ has dense invertibles if $\operatorname{Inv} A$ is dense in $A$. Let $A$ be a commutative, unital Banach algebra, and let $a \in A$. We denote the spectrum of $a$ by $\sigma(a)$ so that $\sigma(a)=\widehat{a}\left(\Phi_{A}\right)$, and we set $\exp A=\{\exp a: a \in A\}$ so that $\sigma(a)=\widehat{a}\left(\Phi_{A}\right)$, and we set $\exp A=\{\exp a: A \in A\}$ so that $\exp A$ is exactly the component of Inv $A$ containing the identity.

Let $X$ be a compact space. A Banach function algebra on $X$ is a unital subalgebra of $C(X)$ that separates the points of $X$ and is a Banach algebra for a norm $\|\cdot\|$; such an algebra is a uniform algebra if it is closed in $\left(C(X),|\cdot|_{X}\right)$. Let $A$ be a Banach function algebra on $X$. As usual, we identify $X$ with the subset of $\Phi_{A}$ consisting of the evaluations at points of $X$, and we say that $A$ is natural if $\Phi_{A}=X$. The Shilov boundary of $A$, denoted by $\Gamma_{A}$, is the minimum (non-empty) closed subset $K$ of $\Phi_{A}$ such that $|f|_{K}=|f|_{\Phi_{A}}(f \in A)$. For $f \in C(X)$, the zero set is

$$
Z_{X}(f)=\{x \in X: f(x)=0\} .
$$

Let $A$ be a commutative, unital Banach algebra. Then $A$ is regular if, for each proper, closed subset $E$ of $\Phi_{A}$ and each $\varphi \in \Phi_{A} \backslash E$, there exists $a \in A$ with $\varphi(a)=1$ and $\psi(a)=0(\psi \in E)$, and $A$ is approximately regular if, for each proper, closed subset $E$ of $\Phi_{A}$ and each $\varphi \in \Phi_{A} \backslash E$, there exists $a \in A$ with $\varphi(a)=1$ and $|\psi(a)|<1(\psi \in E)$. Let $E$ be a closed subset of $\Phi_{A}$. Then $A_{E}$ is the closure in $\left(C(E),|\cdot|_{E}\right)$ of $\{\widehat{a} \mid E: a \in A\}$. We see that $A$ is approximately regular if and only if each such uniform algebra $A_{E}$ is natural on $E$. There are many uniform algebras that are approximately regular, but not regular. For example, let $X$ be a compact subset of $\mathbb{C}$ with empty interior. Then $R(X)$ is always approximately regular $[20$, Proposition 4.7], but need not be regular (see, for example, [11]).

\section{The NEW COUNTER-EXAMPLE}

We are now ready to construct our main example.

The topological boundary of a set $X \subseteq \mathbb{C}^{n}$ is denoted by $\partial X$. We shall use the notation $\underline{z}=(z, w)$ for a typical element of $\mathbb{C}^{2}$.

Let $X$ be a compact subset of $\mathbb{C}^{n}$. The polynomially convex hull and the rationally convex hull of $X$ are denoted by $\widehat{X}$ and $h_{r}(X)$, respectively. The algebras $P(X)$ and $R(X)$ are the uniform closures in $C(X)$ of the set of restrictions to $X$ of 
the polynomials and of the rational functions with poles off $X$, respectively. It is standard that $\Phi_{P(X)}$ and $\Phi_{R(X)}$ can be identified with $\widehat{X}$ and $h_{r}(X)$, respectively. With this identification, $P(\widehat{X})$ and $R\left(h_{r}(X)\right)$ are equal to the sets of Gel'fand transforms of the elements of the algebras $P(X)$ and $R(X)$, respectively.

Theorem 2.1. There exists a compact set $Y \subseteq \partial \overline{\mathbb{D}}^{2}$ in $\mathbb{C}^{2}$ such that $(0,0) \in \widehat{Y}$, and yet $P(Y)$ has dense invertibles. In particular, setting $X=\widehat{Y}$, the uniform algebra $P(X)$ is natural on $X$ and has dense invertibles, but $\Gamma_{P(X)}$ is a proper subset of $X$.

Proof. Let $\mathcal{F}$ be the family of all non-constant polynomials $p$ in two variables with coefficients in $\mathbb{Q}+\mathrm{i} \mathbb{Q}$ such that $p\left(\overline{\mathbb{D}}^{2}\right) \subseteq \overline{\mathbb{D}}$. This is a countable family, and the family $\{p \mid Y: p \in \mathcal{F}\}$ is guaranteed to be dense in the unit ball of $P(Y)$ whenever $Y$ is a compact subset of $\overline{\mathbb{D}}^{2}$. We shall construct a compact set $Y \subseteq \partial \overline{\mathbb{D}}^{2}$ such that $(0,0) \in \widehat{Y}$ and such that, for each $p \in \mathcal{F}$, the spectrum of $p \mid Y$ with respect to $P(Y)$ has empty interior. From this it quickly follows that $Y$ has the desired properties.

Choose a countable, dense subset $\left\{\zeta_{i}: i=1,2, \ldots\right\}$ of $\mathbb{D}$ which does not meet the countable set $\{p(0,0): p \in \mathcal{F}\}$. Define sets $E_{i, p}$ for $i \in \mathbb{N}$ and $p \in \mathcal{F}$ by

$$
E_{i, p}=\left\{\underline{z} \in \overline{\mathbb{D}}^{2}: p(\underline{z})=\zeta_{i}\right\} .
$$

Each $E_{i, p}$ is compact, and there are only countably many such sets. Enumerate those pairs $(i, p) \in \mathbb{N} \times \mathcal{F}$ for which $E_{i, p}$ is non-empty as $\left(i_{j}, p_{j}\right)_{j=1}^{\infty}$, and then set $K_{j}=E_{i_{j}, p_{j}}(j \in \mathbb{N})$. For notational convenience, set $a_{j}=\zeta_{i_{j}}$, so that

$$
K_{j}=\left\{\underline{z} \in \overline{\mathbb{D}}^{2}: p_{j}(\underline{z})=a_{j}\right\} .
$$

Note that there may be repeats in the sequence $\left(a_{j}\right)$, and that the sets $K_{j}$ need not be pairwise disjoint, but this will not matter.

Define polynomials $G_{j}$ for $j \in \mathbb{N}$ by

$$
G_{j}=\frac{p_{j}-a_{j}}{p_{j}(0,0)-a_{j}} .
$$

Then $G_{j} \mid K_{j}=0$ and $G_{j}(0,0)=1$ for each $j \in \mathbb{N}$.

We now define inductively a sequence $\left(F_{j}\right)$ of entire functions on $\mathbb{C}^{2}$ which also satisfy the conditions that $F_{j} \mid K_{j}=0$ and $F_{j}(0,0)=1$ for each $j \in \mathbb{N}$.

We begin with $F_{1}=G_{1}$. Thus $F_{1} \mid K_{1}=0$ and $F_{1}(0,0)=1$.

Now suppose that $j \in \mathbb{N}$, and assume that $F_{1}, \ldots, F_{j}$ have been defined so as to satisfy $F_{m} \mid K_{m}=0$ and $F_{m}(0,0)=1(m=1, \ldots, j)$. Set

$$
L_{j}=\left\{\underline{z} \in \overline{\mathbb{D}}^{2}: \operatorname{Re} F_{j}(\underline{z}) \leq 1 / 2\right\},
$$

and $H_{j+1}(\underline{z})=\exp \left(F_{j}(\underline{z})-1\right)$. We see that $\left|H_{j+1}\right|_{L_{j}} \leq \exp (-1 / 2)<1$ and that $H_{j+1}(0,0)=1$. Choose $N \in \mathbb{N}$ large enough so that

$$
\left|H_{j+1}^{N} G_{j+1}\right|_{L_{j}}<1 / 4 .
$$

Since $G_{j+1} \mid K_{j+1}=0$, we then also have

$$
\left|H_{j+1}^{N} G_{j+1}\right|_{L_{j} \cup K_{j+1}}<1 / 4 .
$$

Set $F_{j+1}=H_{j+1}^{N} G_{j+1}$. Clearly $F_{j+1} \mid K_{j+1}=0$ and $F_{j+1}(0,0)=1$. The inductive construction now proceeds. 
Note that this construction also produces a sequence $\left(L_{j}\right)$ of compact subsets of $\overline{\mathbb{D}}^{2}$ as specified in (1). We have $K_{j} \subseteq \operatorname{int} L_{j}(j \in \mathbb{N}$ ) (here and below the interior is taken in $\overline{\mathbb{D}}^{2}$ ). Moreover, for each $j \in \mathbb{N}$, we have

$$
\left|F_{j+1}\right|_{L_{j} \cup K_{j+1}}<1 / 4,
$$

and hence $L_{j} \cup K_{j+1} \subseteq \operatorname{int} L_{j+1}$.

For each $j \in \mathbb{N}$, consider the variety $W_{j}=\left\{\underline{z} \in \mathbb{C}^{2}: F_{j}(\underline{z})=1\right\}$. Note that $(0,0) \in W_{j}$ and $W_{j} \cap L_{j}=\emptyset$. Set $V_{j}=W_{j} \cap \overline{\mathbb{D}}^{2}$. Since $W_{j}$ is the zero set of an entire function on $\mathbb{C}^{2}$ which vanishes at $(0,0)$, it follows (using, for example, [13, Theorem C5, Chapter I]) that $V_{j} \cap \partial \overline{\mathbb{D}}^{2} \neq \emptyset$.

Finally, set $M_{j}=\left\{\underline{z} \in \overline{\mathbb{D}}^{2}: \operatorname{Re} F_{j}(\underline{z}) \geq 1 / 2\right\}$. We now see that the following three facts hold.

(a) The sets int $L_{j}$ are nested increasing, and

$$
\bigcup_{j=1}^{\infty} K_{j} \subseteq \bigcup_{j=1}^{\infty} \operatorname{int} L_{j} .
$$

(b) We have $V_{j} \subseteq M_{i}(j \geq i)$.

(c) For each $j \in \mathbb{N}$, there is a polynomial $h_{j}$ such that $\left|h_{j}\right|_{M_{j}}<\left|h_{j}(\underline{z})\right|\left(\underline{z} \in K_{j}\right)$. Here (a) and (b) are clear from the definitions and properties above. For (c), note that

$$
\left|\exp \left(-F_{j}\right)\right|_{M_{j}} \leq \exp (-1 / 2)<1=\exp \left(-F_{j}(\underline{z})\right) \quad\left(\underline{z} \in K_{j}\right),
$$

and so we may take $h_{j}$ to be a suitable partial sum of the power series for $\exp \left(-F_{j}\right)$.

We now consider the space of all non-empty, closed subsets of $\overline{\mathbb{D}}^{2}$, with the Hausdorff metric. (For more details on this metric, see, for example, [8, Appendix A.1].) This metric space is compact, so the sequence $\left(V_{j}\right)$ has a convergent subsequence, say $V_{j_{k}} \rightarrow V \subseteq \overline{\mathbb{D}}^{2}$. We see that $(0,0) \in V$ because $(0,0)$ is in all of the sets $V_{j}$. By property (b) above, $V \subseteq M_{i}$ for each $i \in \mathbb{N}$. Finally, as noted above, all of the sets $V_{j}$ have non-empty intersection with $\partial \overline{\mathbb{D}}^{2}$, and so the same is true of $V$. Set $Y=V \cap \partial \overline{\mathbb{D}}^{2}$, a non-empty, compact set. We shall show that this set $Y$ has the desired properties.

We first show that $\widehat{Y}=\widehat{V}$ (and so, in particular, that $(0,0) \in \widehat{Y}$ ). For this it is enough to show that, for every polynomial $p$, we have $|p|_{V}=|p|_{Y}$. Given a polynomial $p$, assume for contradiction that there is a $\underline{z}_{0} \in V \backslash \partial \overline{\mathbb{D}}^{2}$ such that $\left|p\left(\underline{z}_{0}\right)\right|>|p|_{Y}$. Then there are disjoint open sets $U_{1}$ and $U_{2}$ in $\mathbb{C}^{2}$ with

$$
\underline{z}_{0} \in U_{1} \subseteq \mathbb{D}^{2}
$$

with $Y \subseteq U_{2}$, and such that $|p(\underline{z})|>|p|_{U_{2}}$ for all $\underline{z} \in U_{1}$. By the definition of $V$, there must be a $j \in \mathbb{N}$ such that $V_{j} \cap U_{1} \neq \emptyset$ and $V_{j} \cap \partial \overline{\mathbb{D}}^{2} \subseteq U_{2}$. Thus $\max \left\{|p(\underline{z})|: \underline{z} \in V_{j}\right\}$ is attained at a point of $\mathbb{D}^{2}$, and not at any point of $V_{j} \cap \partial \overline{\mathbb{D}}^{2}$. This contradicts the maximum principle [13, Theorem B16, Chapter III] on $V_{j}$. Thus we must have $\widehat{Y}=\widehat{V}$, as claimed.

Finally, let $p \in \mathcal{F}$. We wish to show that the spectrum of $p \mid Y$ in $P(Y)$ has empty interior, i.e., that the set $p(\widehat{Y})=p(\widehat{V})$ has empty interior. Assume for contradiction that this is not the case. Then there must be some $i \in \mathbb{N}$ with $\zeta_{i} \in p(\widehat{V})$, and there is some $\underline{z}_{0} \in \widehat{V}$ with $p\left(\underline{z}_{0}\right)=\zeta_{i}$. In particular, $\underline{z}_{0} \in E_{i, p}$, and so there is a $j \in \mathbb{N}$ 
with $\zeta_{i}=a_{j}, p=p_{j}$, and $E_{i, p}=K_{j}$. Thus $\underline{z}_{0} \in K_{j} \cap \widehat{V}$. Since $V \subseteq M_{j}$, it follows from (c), above, that the polynomial $h_{j}$ satisfies

$$
\left|h_{j}\right|_{V} \leq\left|h_{j}\right|_{M_{j}}<\left|h_{j}\left(\underline{z}_{0}\right)\right|,
$$

and this contradicts the fact that $\underline{z}_{0} \in \widehat{V}$.

We have proved that $Y$ has the desired properties.

Finally, we set $X=\widehat{Y}$. The required properties of $P(X)$ are now immediate from the standard theory discussed earlier.

Remarks. Let $X$ and $Y$ be as in Theorem 2.1, and set $A=P(X)$.

(a) The coordinate projections $\pi_{1}:(z, w) \mapsto z$ and $\pi_{2}:(z, w) \mapsto w$ are polynomials and are clearly in $\mathcal{F}$. Thus the projections of $X$ on the two coordinate planes have empty interior, and so int $X=\emptyset$.

(b) Since $A$ is natural on $X$ and $A \neq C(X)$, we must have $\operatorname{dim} X>0$. By [14, Theorem IV 3], since int $X=\emptyset$, we must have $\operatorname{dim} X \leq 3$. In fact, $P(X)$ clearly has 2 generators, and so, by [7, Remark 2.2], $\operatorname{dim} X \leq 2$. Thus $\operatorname{dim} X$ is equal to either 1 or 2 . We conjecture that $\operatorname{dim} X=2$; if so, as we shall note below, $C(X)$ does not have dense invertibles.

(c) Set $E=\pi_{1}(X)$. Since $E$ has empty interior, the Shilov boundary of $R(E)$ is $E$. By considering functions in $A$ of the form $f:(z, w) \mapsto r(z) p(w)$, where $r \in R(E)$ and $p$ is a polynomial, we see easily that $X \cap(\overline{\mathbb{D}} \times \mathbb{T}) \subseteq \Gamma_{A}$. Applying the same argument to the second coordinate projection, we obtain $X \cap \partial \overline{\mathbb{D}}^{2} \subseteq \Gamma_{A}$, and hence we must have $\Gamma_{A}=Y=X \cap \partial \overline{\mathbb{D}}^{2}$.

The following result shows that the example constructed above has some further unusual properties. This result is probably known, but we know of no explicit reference. We use the term clopen to describe sets which are both open and closed.

Theorem 2.2. Let $A$ be a uniform algebra with compact character space $\Phi_{A}$, and suppose that $A$ has dense invertibles. Then every component of every (non-empty) zero set for $A$ meets $\Gamma_{A}$.

Proof. Set $X=\Gamma_{A}$. Assume for contradiction that $f \in A$ has a non-empty zero set $Z(f)$ which has a component $K \subseteq \Phi_{A} \backslash X$. Then $K$ is the intersection of the family of all relatively clopen subsets of $Z(f)$ which contain $K$, and so there is a relatively clopen subset $E$ of $Z(f)$ with $K \subseteq E \subseteq \Phi_{A} \backslash X$. We may then choose an open subset $U$ of $\Phi_{A} \backslash X$ with $E \subseteq U$ and such that $Y:=\partial U \subseteq \Phi_{A} \backslash Z(f)$.

Set $\delta=\inf \{|f(x)|: x \in Y\}$, so that $\delta>0$, and then choose an invertible element $g \in A$ with $|g-f|_{X}<\delta / 2$. Then $\left|g^{-1}\right|_{K}>2 / \delta>\left|g^{-1}\right|_{Y}$, which contradicts the local maximum modulus principle [17, Theorem 9.8].

Let $X$ and $Y$ be as constructed in Theorem 2.1. Let $\underline{z} \in \widehat{X}$, and suppose that $p$ is a polynomial with $p(\underline{z})=0$. Then, since $P(X)$ has dense invertibles, the zero set of $p$ must meet $Y$. It follows that $\underline{z}$ is in the rational hull $h_{r}(Y)$ of $Y$. Thus we have $h_{r}(Y)=\widehat{Y}$ and $P(Y)=R(Y)$.

From the fact that $(0,0) \in \widehat{Y}=h_{r}(Y)$ we quickly deduce that, for all $a \in \mathbb{C}$, there is some $(z, w) \in Y$ with $z=a w$. Set $U=\{(z, w) \in X: w \neq 0\}$. Then the image of $U$ under the rational function $z / w$ is the whole of $\mathbb{C}$. This suggests that the set $X$ is fairly large, but does not by itself contradict the possibility that $\operatorname{dim} X=1$. 
We conclude this section with another example with some even stronger properties.

Theorem 2.3. There is a uniform algebra $A$ on a compact metric space such that every point of $\Phi_{A}$ is a one-point Gleason part and such that the invertible elements are dense in $A$, but $\Gamma_{A} \neq \Phi_{A}$.

Proof. Taking the example above as our base algebra, we may form a system of root extensions (as in $[3]$ and $[17, \S 19]$ ) to obtain a new uniform algebra $A$ on a compact metric space such that $\left\{f^{2}: f \in A\right\}$ is dense in $A$, and hence every point of $\Phi_{A}$ is a one-point Gleason part. Since the base algebra has proper Shilov boundary, the same is true for $A$ [3]. Finally, since the base algebra has dense invertibles, so does $A[9$, pp. 2837-2838].

\section{TOPOLOGICAL STABLE RANK}

We now discuss the topological stable rank and some related ranks of a commutative Banach algebra. Indeed, there is a variety of different types of stable rank; for more details, history and open questions, see, for example, [2, 7, 15].

Let $A$ be a commutative, unital Banach algebra, and let $a_{1}, \ldots, a_{n} \in A$. Set $a=\left(a_{1}, \ldots, a_{n}\right) \in A^{n}$. Then the joint spectrum of $a$ is denoted by $\sigma(a)$, so that $\sigma(a)=\widehat{a}\left(\Phi_{A}\right)$. The element $a$ is unimodular if $\sum_{i=1}^{n} A a_{i}=A$; we denote the set of unimodular elements of $A^{n}$ by $U_{n}(A)$. Thus $a \in U_{n}(A)$ if and only if $0 \notin \sigma(a)$ if and only if $\widehat{a_{1}}, \ldots, \widehat{a_{n}}$ have no common zero on $\Phi_{A}$.

We begin with the definitions of the (Bass) stable rank and topological stable rank, given just for commutative Banach algebras.

Definition 3.1. Let $A$ be a commutative, unital Banach algebra.

The (Bass) stable rank of $A$ is the least $n \in \mathbb{N}$ with the property that, for all $\left(a_{1}, a_{2}, \ldots, a_{n+1}\right) \in U_{n+1}(A)$, there exists $\left(b_{1}, b_{2}, \ldots, b_{n}\right) \in A^{n}$ such that

$$
\left(a_{1}+b_{1} a_{n+1}, a_{2}+b_{2} a_{n+1}, \ldots, a_{n}+b_{n} a_{n+1}\right) \in U_{n}(A),
$$

or $\infty$ if no such $n$ exists.

The topological stable rank of $A$ is the least $n \in \mathbb{N}$ such that $U_{n}(A)$ is dense in $A^{n}$, or $\infty$ if no such $n$ exists.

The stable rank and the topological stable rank of $A$ are denoted by $\operatorname{sr}(A)$ and $\operatorname{tsr}(A)$, respectively.

We see immediately that a commutative, unital Banach algebra $A$ has dense invertibles if and only if $\operatorname{tsr}(A)=1$.

We now quote some standard results concerning (topological) stable ranks.

Let $A$ be a commutative, unital Banach algebra. For each $n \in \mathbb{N}$, we have $\operatorname{sr}(A) \leq n$ if and only if the natural map $U_{n}(A) \rightarrow U_{n}(A / I)$ induced by the natural projection $A \rightarrow A / I$ is a surjection for every closed ideal $I$ in $A$ [4, Theorem 4]. Further,

$$
\operatorname{sr}(A) \leq \operatorname{tsr}(A)
$$

this is [4, Theorem 3] and [15, Theorem 2.3]. This inequality is strict for the disk algebra $A$, where $\operatorname{sr}(A)=1$ and $\operatorname{tsr}(A)=2[5,6]$.

Let $X$ be a compact space, and $\operatorname{set} d=\operatorname{dim} X$. Then

$$
\operatorname{sr}(C(X))=\operatorname{tsr}(C(X))=[d / 2]+1,
$$


where $[t]$ denotes the greatest integer less than or equal to $t[21]$. In particular, $C(X)$ has dense invertibles if and only if $\operatorname{dim} X \in\{0,1\}$.

Let $A$ be a commutative, unital Banach algebra. Then

$$
\operatorname{sr}(A) \leq \operatorname{sr}\left(C\left(\Phi_{A}\right)\right) .
$$

This is [6, Corollary 1.6]; it depends on a deep generalization of the Arens-Royden theorem given in [18]. This inequality is strict in many cases; for example, if $A$ is the disk algebra, then $\operatorname{sr}(A)=1$, whereas $\operatorname{sr}(C(\overline{\mathbb{D}}))=2$.

Now let $A$ be a regular Banach function algebra on $X=\Phi_{A}$. Then it is proved in $[6$, Corollary 1.5$]$ that

$$
\operatorname{sr}(A) \geq \operatorname{sr}(C(X)),
$$

and so, by $(4), \operatorname{sr}(A)=\operatorname{sr}(C(X))$. It then follows from (2), (3), and (5) that

$$
\operatorname{tsr}(A) \geq \operatorname{tsr}\left(C\left(\Phi_{A}\right)\right) .
$$

We shall strengthen this last result by giving an elementary proof that applies to approximately regular, rather than regular, algebras. This proof uses the standard version of the Arens-Royden theorem [12, Theorem 7.2].

We first give a topological lemma.

Lemma 3.2. Let $X$ be a compact space, let $E$ be a closed subset of $X$, and let $n \in \mathbb{N}$. Suppose that $\left(f_{1}, \ldots, f_{n}\right) \in C(E)^{n}$, that $\left(h_{1}, \ldots, h_{n}\right) \in U_{n}(C(X))$, and that there exist open subsets $U_{1}, \ldots, U_{n}$ of $X$ satisfying the following properties:

(i) $\bigcup_{i=1}^{n} U_{i}=X$;

(ii) $0 \notin h_{i}\left(U_{i}\right)(i=1, \ldots, n)$;

(iii) $h_{i}\left|E \cap U_{i}=f_{i}\right| E \cap U_{i}(i=1, \ldots, n)$.

Then there exists $\left(g_{1}, \ldots, g_{n}\right) \in U_{n}(C(X))$ such that $g_{i} \mid E=f_{i}(i=1, \ldots, n)$.

Proof. Choose closed subsets $X_{1}, \ldots, X_{n}$ of $X$ such that $X_{i} \subseteq U_{i}(i=1, \ldots, n)$ and $\bigcup_{i=1}^{n} X_{i}=X$, and then, for $i=1, \ldots, n$, define $g_{i}$ on $X_{i} \cup E$ so that $g_{i} \mid E=f_{i}$ and $g_{i}\left|X_{i}=h_{i}\right| X_{i}$. Clearly $g_{i} \in C\left(X_{i} \cup E\right)$. Next extend $g_{i}$ to a function, also called $g_{i}$, in $C(X)$. We note that, by (ii), $Z_{X}\left(g_{i}\right) \cap X_{i}=\emptyset(i=1, \ldots, n)$. Then $\left(g_{1}, \ldots, g_{n}\right)$ has the required properties.

Theorem 3.3. Let $A$ be an approximately regular, commutative, unital Banach algebra. Then $\operatorname{tsr}(A) \geq \operatorname{tsr}\left(C\left(\Phi_{A}\right)\right)$.

Proof. We may suppose that $\operatorname{tsr}(A)<\infty$; set $n=\operatorname{tsr}(A)$. Recall that we have $\operatorname{sr}\left(C\left(\Phi_{A}\right)\right)=\operatorname{tsr}\left(C\left(\Phi_{A}\right)\right)$. We shall show that $\operatorname{sr}\left(C\left(\Phi_{A}\right)\right) \leq n$.

Let $I$ be a closed ideal in $C\left(\Phi_{A}\right)$. Then we can identify $C\left(\Phi_{A}\right) / I$ with $C(E)$ for a certain closed subset $E$ of $X:=\Phi_{A}$. Let $\left(f_{1}, \ldots, f_{n}\right) \in U_{n}(C(E))$, so that $\bigcap_{i=1}^{n} Z_{E}\left(f_{i}\right)=\emptyset$. For $i=1, \ldots, n$, choose closed subsets $F_{1}, \ldots, F_{n}$ of $X$ such that $F_{i} \cap Z_{E}\left(f_{i}\right)=\emptyset(i=1, \ldots, n)$ and $X=\bigcup_{i=1}^{n} \operatorname{int}_{X} F_{i}$. Set

$$
E_{i}=E \cap F_{i} \quad(i=1, \ldots, n) .
$$

Let $i \in\{1, \ldots, n\}$. Since $A$ is approximately regular, $A_{E_{i}}$ is a natural Banach function algebra on $E_{i}$, and so, by the standard Arens-Royden theorem, there exist $b_{i} \in \operatorname{Inv}\left(A_{E_{i}}\right)$ and $r_{i} \in C\left(E_{i}\right)$ with $f_{i}=b_{i} \exp r_{i}$ in $C\left(E_{i}\right)$. Since $\operatorname{Inv}\left(A_{E_{i}}\right)$ is open in $A_{E_{i}}$, we may suppose that $b_{i}=\widehat{a_{i}} \mid E_{i}$ for some $a_{i} \in A$. Since $U_{n}(A)$ is dense in 
$A^{n}$, we may further suppose that $\left(a_{1}, \ldots, a_{n}\right) \in U_{n}(A)$. We may also extend $r_{i}$ so that $r_{i} \in C(X)$, whilst preserving the equalities

$$
f_{i}\left|E_{i}=\left(b_{i} \exp r_{i}\right)\right| E_{i}=\left(\widehat{a_{i}} \exp r_{i}\right) \mid E_{i} .
$$

Set $h_{i}=\widehat{a_{i}} \exp r_{i} \in C(X)(i=1, \ldots, n)$. Then $\left(h_{1}, \ldots, h_{n}\right) \in U_{n}(C(X))$, and $h_{i}\left|E_{i}=f_{i}\right| E_{i}(i=1, \ldots, n)$. For each $i \in\{1, \ldots, n\}$, define $H_{i}$ to be the closure of the set

$$
\left\{x \in E: f_{i}(x) \neq h_{i}(x)\right\},
$$

so that $\bigcap_{i=1}^{n} H_{i}=\emptyset$ by the choice of $F_{1}, \ldots, F_{n}$. Set

$$
U_{i}=X \backslash\left(Z_{X}\left(h_{i}\right) \cup H_{i}\right) \quad(i=1, \ldots, n),
$$

so that each $U_{i}$ is an open subset of $X$.

We verify that clauses (i), (ii), and (iii) of Lemma 3.2 are satisfied. Clearly we have $0 \notin h_{i}\left(U_{i}\right)$ and $h_{i}\left|E \cap U_{i}=f_{i}\right| E \cap U_{i}$ for each $i \in\{1, \ldots, n\}$.

To show that $\bigcup_{i=1}^{n} U_{i}=X$, we must see that $S:=\bigcap_{i=1}^{n}\left(Z_{X}\left(h_{i}\right) \cup H_{i}\right)=\emptyset$. Clearly $S \subseteq E$ because $\bigcap_{i=1}^{n} Z_{X}\left(h_{i}\right)=\emptyset$. Assume that $x \in S$, say

$$
x \in E \cap \operatorname{int}_{X} F_{i} \subseteq \operatorname{int}_{E} E_{i}
$$

for some $i \in\{1, \ldots, n\}$. We have $x \in Z_{X}\left(h_{i}\right) \cup H_{i}$. Since $\left(\operatorname{int}_{E} E_{i}\right) \cap H_{i}=\emptyset$, we have $x \notin H_{i}$. Thus $h_{i}(x)=0$. Since $h_{i}\left|E_{i}=f_{i}\right| E_{i}$, we have $f_{i}(x)=0$. However $Z_{E}\left(f_{i}\right) \cap E_{i}=\emptyset$, and so this is the required contradiction.

It follows from Lemma 3.2 that there exists $\left(g_{1}, \ldots, g_{n}\right) \in U_{n}(C(X))$ such that $g_{i} \mid E=f_{i}(i=1, \ldots, n)$. This shows that $\operatorname{sr}(C(X)) \leq n$, as required.

Corollary 3.4. Let $A$ be an approximately regular, commutative, unital Banach algebra with dense invertibles. Then $C\left(\Phi_{A}\right)$ has dense invertibles.

\section{Open questions}

We conclude with some open questions.

(1) Let $X$ be as constructed in Theorem 2.1, and set

$$
M=\{f \in P(X): f(0,0)=0\} .
$$

What can be said about $M^{2}$ and $\overline{M^{2}}$ ?

(2) Let $A$ be a uniform algebra with the property that $\exp A$ is dense in $A$. Can the Shilov boundary of $A$ be proper? Must $A$ be approximately regular? Must $A$ be $C(X)$ ?

It is conceivable (but unlikely) that $\exp A$ is dense in $A$ for the example $A=P(X)$ of Theorem 2.1. This is true if and only if $\exp A=\operatorname{Inv} A$, because $A$ has dense invertibles. By [12, Chapter III, Corollary 7.4], $\exp A=\operatorname{Inv} A$ if and only if $H^{1}(X, \mathbb{Z})$, the first Cech cohomology group of $X$ with integer coefficients, is trivial. Thus, for our example, $\exp A$ is dense in $A$ if and only if $X$ is simply coconnected in the sense of [17, Definition 29.24].

(3) Let $A$ be a uniform algebra with character space $X$. Suppose that $C(X)$ has dense invertibles. Must $A$ have dense invertibles? Does it help to assume that $A$ is regular/approximately regular/has Shilov boundary $X$ ? More generally (as asked in [7]), is it always true that $\operatorname{tsr}(A) \leq \operatorname{tsr}(C(X))$, and hence $\operatorname{tsr}\left(C\left(\Phi_{A}\right)\right)=\operatorname{tsr}(A)$ in the case where $A$ is approximately regular?

We offer the following caution. Assume that one can prove that $A$ has dense invertibles whenever $A$ is approximately regular and $C\left(\Phi_{A}\right)$ has dense 
invertibles. Then we would have a solution to the famous 'Gel'fand problem': Is there a natural uniform algebra $A$ on $\mathbb{I}$ such that $A \neq C(\mathbb{I})$ ? Indeed, suppose that $A$ is a natural uniform algebra on $\mathbb{I}$. By [20, Lemma 3.1], $A$ is approximately regular. By our assumption, $A$ has dense invertibles, and so $A=C(\mathbb{I})$ by [9, Corollary 1.8].

(4) Let $A$ be a uniform algebra with dense invertibles. Does it follow that $C\left(\Phi_{A}\right)$ has dense invertibles? More generally (as asked in [7]), must we have $\operatorname{tsr}\left(C\left(\Phi_{A}\right)\right) \leq \operatorname{tsr}(A)$ ?

Let $X$ be as constructed in Theorem 2.1, and set $A=P(X)$. We have conjectured that $\operatorname{dim} X=2$. If so, then we would have a negative answer to the first question because $C\left(\Phi_{A}\right)$ would not have dense invertibles, but $A$ does.

(5) Let $A$ be an approximately regular, commutative, unital Banach algebra. Is it true that $\operatorname{sr}(A) \geq \operatorname{sr}\left(C\left(\Phi_{A}\right)\right)$ ?

(6) Our final questions concern the existence of topological disks in the character space of uniform algebras.

(a) Let $A$ be a uniform algebra such that $\Gamma_{A} \neq \Phi_{A}$. Does $\Phi_{A} \backslash \Gamma_{A}$ contain a homeomorphic copy of $\overline{\mathbb{D}}$ ?

(b) Let $K$ be a compact subset of $\mathbb{C}^{n}$ such that $\widehat{K} \neq K$. Does $\widehat{K} \backslash K$ contain a homeomorphic copy of $\overline{\mathbb{D}}$ ?

We would like to thank the referee for valuable comments and, in particular, for a helpful suggestion that led to the present version of Theorem 3.3.

\section{REFERENCES}

[1] H. Alexander and J. Wermer, Several complex variables and Banach algebras, Graduate Texts in Mathematics, 35, Springer-Verlag, New York, 1998. MR1482798 (98g:32002)

[2] C. Badea, The stable rank of topological algebras and a problem of R. G. Swan, J. Functional Analysis, 160 (1998), 42-78. MR1658716 (2000a:46069)

[3] B. J. Cole, One point parts and the peak point conjecture, Ph.D. Thesis, Yale University, 1968.

[4] G. Corach and A. R. Larotonda, Stable rank in Banach algebras, Journal of Pure and Applied Algebra, 32 (1984), 289-300. MR745359 (86g:46070)

[5] G. Corach and F. D. Suárez, Stable rank in holomorphic function algebras, Illinois J. Math., 29 (1985), 627-639. MR806470 (87b:46056)

[6] G. Corach and F. D. Suárez, Extension problems and stable rank in commutative Banach algebras, Topology and its applications, 21 (1985), 1-8. MR808718 (87a:46086)

[7] G. Corach and F. D. Suárez, Thin spectra and stable range conditions, J. Functional Analysis, 81 (1988) 432-442. MR971887 (89k:46061)

[8] H. G. Dales, Banach algebras and automatic continuity, London Mathematical Society Monographs, New Series, Volume 24, The Clarendon Press, Oxford, 2000. MR1816726 (2002e:46001)

[9] T. W. Dawson and J. F. Feinstein, On the denseness of the invertible group in Banach algebras, Proc. American Math. Soc., 131 (2003), 2831-2839. MR1974340 (2004c:46093)

[10] C. M. Falcón Rodríguez, The denseness of the group of invertible elements of a uniform algebra, Cienc. Mat. (Havana), 9 (1988), 11-17. MR1007646 (91c:46072)

[11] J. F. Feinstein, Trivial Jensen measures without regularity, Studia Mathematica, 148 (2001), 67-74. MR1881440 (2002k:46127)

[12] T. W. Gamelin, Uniform algebras, Prentice-Hall, Englewood Cliffs, New Jersey, 1969. MR0410387 (53:14137)

[13] R. C. Gunning and H. Rossi, Analytic functions of several complex variables, Prentice-Hall, Englewood Cliffs, New Jersey, 1965. MR0180696 (31:4927)

[14] W. Hurewicz and H. Wallman, Dimension theory, Princeton University Press, Princeton, New Jersey, 1948. MR0006493 (3:312b) 
[15] M. A. Rieffel, Dimension and stable rank in the $K$-theory of $\mathrm{C}^{*}$-algebras, Proc. London Math. Soc., 46 (1983), 301-333. MR693043 (84g:46085)

[16] G. Stolzenberg, A hull with no analytic structure, J. Mathematics and Mechanics, 12 (1963), 103-111. MR0143061 (26:627)

[17] E. L. Stout, The theory of uniform algebras, Bogden and Quigley, New York, 1971. MR0423083 (54:11066)

[18] J. L. Taylor, Topological invariants of the maximal ideal space of a Banach algebra, Advances in Mathematics, 19 (1976), 149-206. MR0410384 (53:14134)

[19] J. Wermer, On an example of Stolzenberg, Symposium on Several Complex Variables (Park City, Utah, 1970), Lecture Notes in Math., Vol. 184, Springer, Berlin, 1971, pp. 79-84. MR0298428 (45:7480)

[20] D. R. Wilken, Approximate normality and function algebras on the interval and circle, in Function algebras, (ed. F. T. Birtel), Scott, Foresman, Glenview, Illinois, 1965, pp. 98-111. MR0196525 (33:4712)

[21] L. N. Vaserstein, Stable rank of rings and dimensionality of topological spaces, Functional Analysis and Applications, 5 (1972), 102-110.

Department of Pure Mathematics, University of Leeds, Leeds LS2 9JT, United KingDOM

E-mail address: garth@maths.leeds.ac.uk

School of Mathematical Sciences, University of Nottingham, Nottingham NG7 2RD, UNITED Kingdom

E-mail address: Joel.Feinstein@nottingham.ac.uk 